\title{
DEVELOPMENT OF AN OPTICAL TRANSITION RADIATION DETECTOR FOR PROFILE MONITORING OF ANTIPROTON AND PROTON BEAMS AT FNAL *
}

\author{
V. E. Scarpine", C. W. Lindenmeyer, G. R. Tassotto, FNAL, Batavia, IL 60510, U.S.A. \\ A. H. Lumpkin, ANL, Argonne, IL 60439, U.S.A.
}

\begin{abstract}
Optical transition radiation (OTR) detectors are being developed at Fermi National Accelerator Laboratory (FNAL) as part of the collider Run II upgrade program and as part of the NuMI primary beam line. These detectors are designed to measure $150 \mathrm{GeV}$ antiprotons as well as $120 \mathrm{GeV}$ proton beams over a large range of intensities. Design and development of an OTR detector capable of measuring beam in both directions down to beam intensities of $\sim 5 \mathrm{e} 9$ particles for nominal beam sizes are presented. Applications of these OTR detectors as an on-line emittance monitor for both antiproton transfers and reverse-injected protons, as a Tevatron injection profile monitor, and as a high-intensity beam profile monitor for NuMI are discussed. In addition, different types of OTR foils are being evaluated for operation over the intensity range of $\sim 5 \mathrm{e} 9$ to $5 \mathrm{e} 13$ particles per pulse, and these are described.
\end{abstract}

\section{INTRODUCTION}

Particle-beam diagnostic techniques based on optical transition radiation (OTR) have been demonstrated at a number of facilities over a wide range of beam energy (or Lorentz factor, $\gamma$ ) [1] - [3]. Optical transition radiation is generated when a charged particle transits the interface between two media with different dielectric constants [2]. This radiation is emitted over the visible spectrum; so, optical imaging techniques can be used to acquire the OTR signal and then reconstruct beam size and position at the dielectric surface. Since OTR is a surface phenomenon, thin foils are used as the converter to reduce beam scattering and minimize heat deposition.

FNAL is developing an OTR detector for the Tevatron collider (Run II) and for high-intensity proton beams for neutrino experiments (NuMI). These detectors will be used to measure beam properties including transverse profiles and 2-D shape, transverse position, divergence, emittance and intensity. These detectors will be installed in (1) the A150 transport line between the Main Injector and the Tevatron to measure protons and antiprotons, (2) the Tevatron ring for injection study measurements adjacent to the new Tevatron Ionization Profile Monitor (IPM) and (3) the NuMI transport line just upstream of the primary target. These three locations require the OTR detector to operate over a large range of beam conditions. Table 1 gives a summary of the relevant beam conditions for our OTR detector design.

Construction and operation of a prototype OTR detector

*Work supported by the U. S. Department of Energy Contracts No. DEAC02-CH03000 and W-31-109-ENG-38.

"scarpine@fnal.gov at FNAL demonstrated the feasibility of using an OTR detector to measure intense proton beams [4] - [6]. This prototype detector measured OTR signal for $120 \mathrm{GeV}$ protons with intensities up to $4.7 \times 10^{12}$ particles in a $1.6 \mu \mathrm{s}$ spill at a rate of one spill every 2 seconds.

Table 1: Beam Parameters for OTR Detector Locations

\begin{tabular}{|l|c|c|c|}
\hline & A150 Line & Tevatron & NuMI \\
\hline Beam Type & $\begin{array}{c}\text { Proton/ } \\
\text { Antiproton }\end{array}$ & $\begin{array}{c}\text { Proton/ } \\
\text { Antiproton }\end{array}$ & Proton \\
\hline $\begin{array}{l}\text { Beam Energy } \\
(\mathrm{GeV})\end{array}$ & 150 & 150 & 120 \\
\hline $\begin{array}{l}\text { Beam Intensity } \\
(\text { E10) }\end{array}$ & $\sim 1$ to 50 & $\sim 1$ to 50 & $\begin{array}{c}\sim 10 \text { to } \\
5000\end{array}$ \\
\hline $\begin{array}{l}\text { Transverse } \\
\begin{array}{l}\text { Beam Size }(\sigma \text { in } \\
\text { mm) }\end{array}\end{array}$ & $\sim 1$ to 4 & $\sim 1.5$ & $\sim 1.0$ \\
\hline Rep Rate & $\begin{array}{c}\sim 10 \text { transfers } \\
\text { per day }\end{array}$ & $\begin{array}{c}\text { studies } \\
\text { only }\end{array}$ & $\begin{array}{c}\sim 0.5 \\
\mathrm{~Hz}\end{array}$ \\
\hline
\end{tabular}

\section{OTR DETECTOR DESIGN}

Figure 1 shows a block diagram of the OTR detector designed for the FNAL proton and antiproton transport lines.

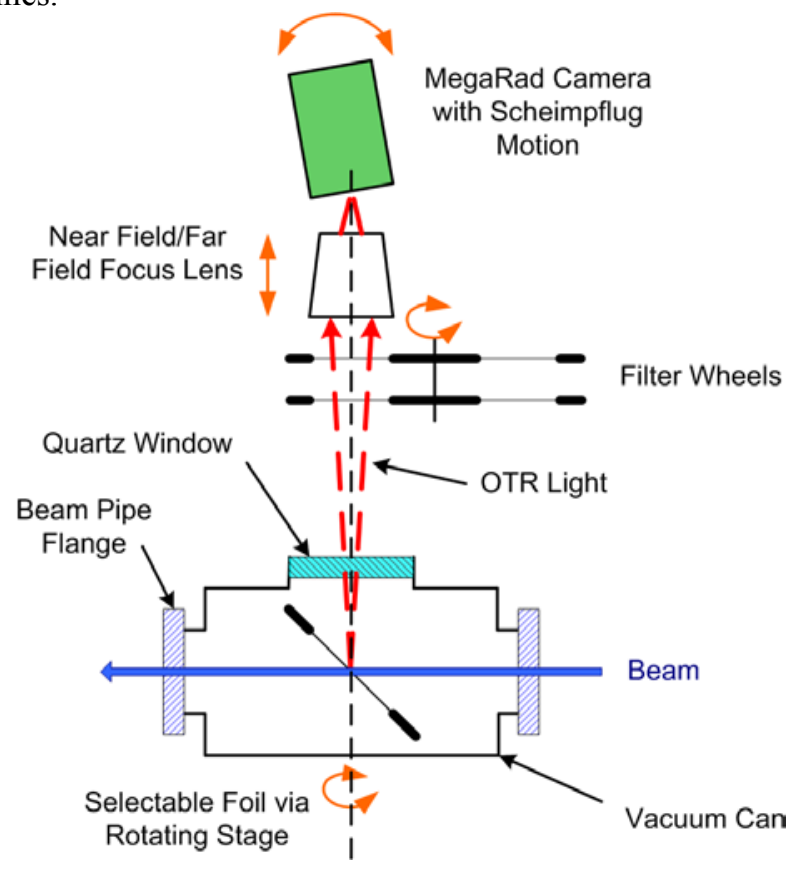

Figure 1: Diagram of OTR detector. 


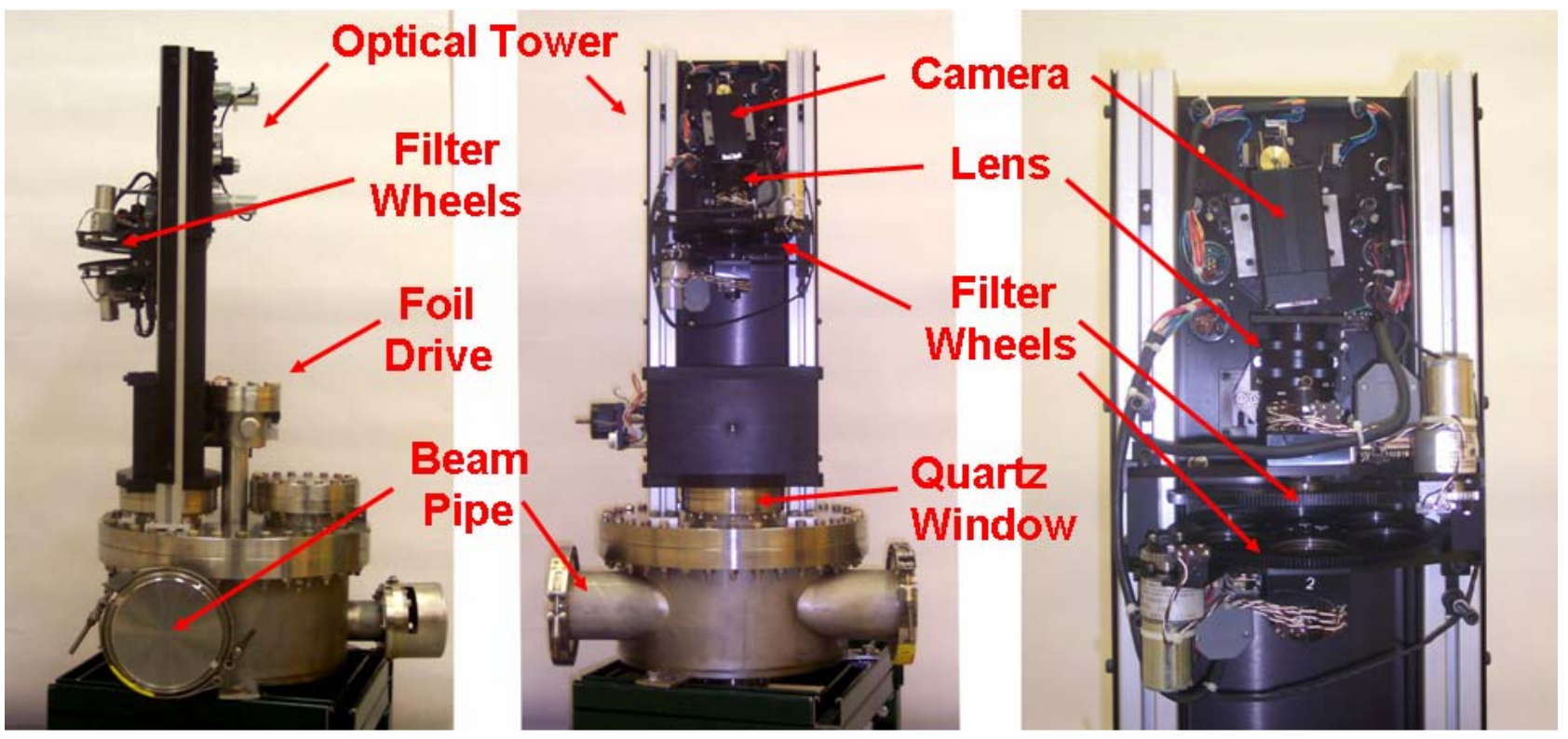

Figure 2: The OTR detector. Left image shows beam line view of detector. Center image shows side of detector. Right image shows magnified view of optical tower with camera, lens and filter wheel assembly.

The design and construction of a single OTR detector for the range of beam conditions required the inclusion of various features. The detector has utilized a two-foil design to allow for bidirectional beam measurements by inserting one of the two foils into the beam. For NuMI, both foils are oriented for a single beam direction. Because of NuMI's high-intensity beam, one foil will be used for operations and the second foil will be used to track changes to the detector response [6]. The mounting of the foils gives a $3 \frac{1}{2}$ inch clear aperture for the beam passage.

A $60 \mathrm{~mm}$ focal length multi-element lens is used to collect the OTR signal and image the beam spot onto the camera. The field of view of the optical system is $\sim 65 \mathrm{x}$ $50 \mathrm{~mm}$ around the beam pipe center. The lens focus can be remotely set to image the beam spot (near field) or set to infinite focus to image the OTR angular distribution (far field). In order to maintain focus over the foils, the camera can be remotely rotated to the corresponding Scheimpflug condition [7]. A set of neutral density filter wheels are used to adjust the signal level to accommodate over three orders of magnitude of OTR. One of the filter wheels incorporates polarizing filters that will be used to measure the orthogonal components of the OTR radial polarization.

Because of the high-energy hadron environment, a radiation hardened Charge Injection Device (CID) camera is used to acquire the OTR signal. The camera is operational up to a MRad $[6,8]$ and can be remotely synchronized to the beam arrival time.

A number of different types of foil materials have been investigated for these detectors. The foils must have minimal effect on the beam but be able to withstand a high-energy hadron beam [6]. A foil of stretched $5 \mu \mathrm{m}$ aluminized mylar will be used for the A150 line and Tevatron OTR detectors. A foil of stretched $6 \mu \mathrm{m}$ aluminized kapton will be used for the NuMI detector. While the mylar foil exhibits optical quality flatness, calculations indicate that kapton will survive better in the intense NuMI beam and provides adequate imaging performance.

Figure 2 shows pictures of a complete OTR detector. This detector has been calibrated and vacuum certified to $\sim 5 \times 10^{-9}$ torr.

\section{DETECTOR CALIBRATION}

A number of calibration steps are taken in order to properly set up and operate the OTR detectors. Mechanical fabrication and assembly of the OTR detector sets the optical axis of the imaging system to intersect the center of the beam pipe. An optical autocollimator set at the beam pipe axis is used to set the optical axis and the foil positions.

After setting the foil positions and aligning the optical axis, the camera focus is set with a focus target placed at each foil position. Figure 3 shows a photograph of the focus target along with an image of the target taken with the OTR detector.

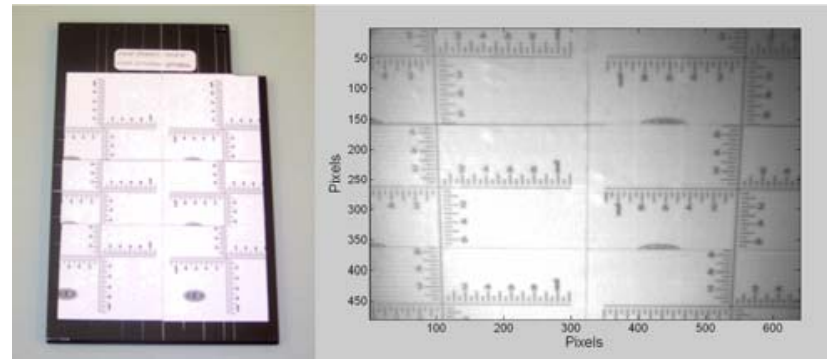

Figure 3: Left image shows photograph of target used to set detector optical focus. Right image is of focus target taken with OTR detector. 
After the camera focus is set, the optical distortion is measured using a target of orthogonal lines. The slopes of the foils produce a perspective distortion in the OTR image, which must be corrected. Figure 4 shows the distortion target and its image taken with the OTR detector.
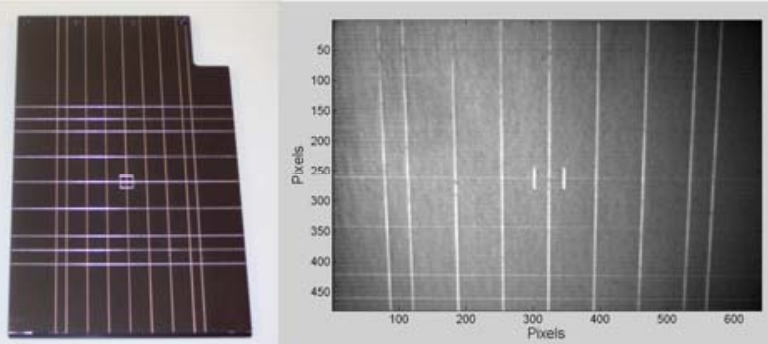

Figure 4: Left image shows photograph of target used to measure OTR detector optical distortion. Right image is of distortion target taken with OTR detector.

In order to allow calibration of the OTR detector insitu, a set of fiducial holes are placed in each foil. These foils are back illuminated to allow adequate imaging of the aluminized foils. Figure 5 show a photograph of an aluminized mylar foil and it's image taken with the OTR detector with the fiducial illumination on.
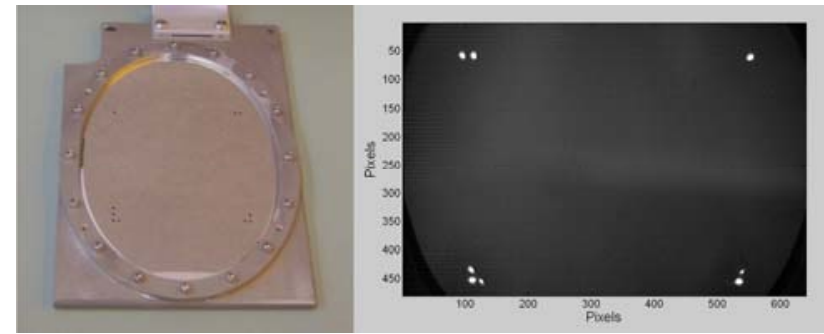

Figure 5: Left image shows photograph of aluminized mylar foil. Right image is of foil taken with OTR detector showing back illuminated fiducial holes.

\section{CONCLUSION}

Results from a prototype OTR detector for highintensity protons indicate that an OTR detector can be built for proton and antiproton beam measurements at FNAL. We have designed, constructed, calibrated and vacuum certified an OTR detector to measure proton and antiproton beams under a range of operating parameters. The detector has been designed to allow for bidirectional beam measurements over more than three orders of magnitude in beam intensity.

\section{ACKNOWLEDGEMENTS}

We thank our colleagues in the FNAL Accelerator Division for their contributions: Eugene Lorman, Jim Fitzgerald, Jim Morgan, Tony Leveling and Elvin Harms. In addition, we wish to thank Jerry Zimmerman, John Korienek, Ron Miksa, Karen Kephart, Eileen Hahn and Wanda Newby of FNAL Particle Physics Division for their outstanding work in the mechanical design and construction of the detector. The authors also acknowledge the support of Gianfranco Ferioli (CERN) and Roland Jung (CERN) for conversations on OTR detectors. We would also like to thank Stephen Pordes (FNAL) and Bob Webber (FNAL) for their enthusiasm and encouragement for pursuing OTR detectors.

\section{REFERENCES}

[1] P. Goldsmith and J. V. Jelley, "Optical Transition Radiation from Protons Entering Metal Surfaces," Phil. Mag., 4, 836844 (1959).

[2] J. Bosser, J. Mann, G. Ferioli and L. Wartski, "Optical Transition Radiation Proton Beam Profile Monitor," CERN/SPS 84-17.

[3] A. H. Lumpkin, B. X. Yang, W. J. Berg, M. White, J. W. Lewellen and S. V. Milton, "Optical Techniques for Electron-Beam Characterizations on the APS SASE FEL Project," Nucl. Instrum. Methods, vol. A429, 336-340 (1999).

[4] A. H. Lumpkin and V. Scarpine, "The Feasibility of OTR Imaging of High-Intensity Proton Beams at FNAL," presented at the Particle Accelerator Conference, Portland, Oregon, May 12-16, 2003.

[5] V. E. Scarpine, A. H. Lumpkin, W. Schappert and G. R. Tassotto, "Optical Transition Radiation Imaging of Intense Proton Beams at FNAL," IEEE Trans. Nucl. Sci.,vol 51, 1529-1532 (2004)

[6] V. E. Scarpine, G. R. Tassotto and A. H. Lumpkin, "Proposed OTR Measurements of $120-\mathrm{GeV}$ Protons and AntiProtons at FNAL," AIP Conference Proceedings 732, 413-419 (2004)

[7] J. Carbone, J. Zarnowski, M. Pace, S. Czebiniak and R. Carta, "Megarad and Scientific CIDs," Proceedings of SPIE, 2654, 131-138 (1996).

[8] W. J. Smith, Modern Optical Engineering, $2^{\text {nd }}$ ed. (Boston, MA: McGraw-Hill, 1990, p. 52). 